\title{
Mass-Like Fat at the Medial Midfoot: A Common Pseudolesion
}

\author{
Colin D. Strickland ${ }^{1} \quad$ Luke W. Patten ${ }^{2}$ Michael J. Durst ${ }^{1} \quad$ Alexander N. Merkle ${ }^{1}$ \\ ${ }^{1}$ Department of Radiology, University of Colorado School of \\ Medicine, Aurora, Colorado, United States \\ ${ }^{2}$ Department of Biostatistics and Informatics, Colorado School of \\ Public Health, Aurora, Colorado, United States

\begin{abstract}
Address for correspondence Colin D. Strickland, MD, Department of Radiology, University of Colorado School of Medicine, Leprino Building, 12401 east 17th Avenue, Aurora, CO 80045, United States (e-mail: colin.strickland@cuanschutz.edu).
\end{abstract}

Indian J Radiol Imaging 2021;31:245-251.

\begin{abstract}
Background Fatty masses are common and may be encountered in the foot and ankle. In some cases, normal subcutaneous fat may be mistaken for a discrete mass. Aims The aim of this study was to evaluate the common finding of prominent subcutaneous fat at the medial midfoot resembling a lipoma and to determine the prevalence of this pseudolesion by applying a series of potential size cutoff criteria.

Materials and Methods Three musculoskeletal radiologists retrospectively evaluated 91 sequentially performed magnetic resonance imaging examinations of the ankle to measure fat resembling a discrete lipoma at the medial midfoot. Each blinded reader measured the largest area of continuous subcutaneous fat in orthogonal axial, coronal craniocaudal, and coronal transverse dimensions. Patient age, sex, and study indications were also recorded. Statistical analysis was performed with $R$ and SAS 9.4 software

Results A discrete fatty pseudolesion as defined by measuring at least $1 \mathrm{~cm}$ in all planes by measurements of at least two of three readers was present in $87 \%$ of cases (79 of 91). When a size criterion of $1.5 \mathrm{~cm}$ was used, a pseudolesion was documented in $14 \%$ of cases (13 of 91). There was a significant correlation between larger pseudolesion size and female sex in the axial plane; however, there was no correlation in the

Keywords

- lipoma

- midfoot

- mass coronal craniocaudal and coronal transverse dimensions.

Conclusions Subcutaneous fat at the medial midfoot often has a mass-like appearance that could be mistaken for a lipoma. It is important to recognize this pseudolesion variant and not to confuse the imaging appearance for a discrete mass.
\end{abstract}

\section{Introduction}

Lipomas are common benign neoplasms found throughout the body. Soft-tissue lipomas typically present as a soft mobile mass and are often clinically diagnosed, escaping imaging evaluation. By magnetic resonance imaging (MRI), they are readily identified by their fatty content and may be discovered incidentally during evaluation for

published online

July 28,2021
DOI https://doi.org/

$10.1055 / \mathrm{s}-0041-1732826$ ISSN 0971-3026 another unrelated indication. Lipomas are the most common soft-tissue neoplasm and account for a large proportion of soft tissue masses reported in the medical literature. ${ }^{1,2}$ They are more common in women and increase in prevalence with advancing age. ${ }^{1}$

When evaluating a mass, it is critical to distinguish lipoma from liposarcoma or other malignant lesions that may be encountered in the soft tissues. Typical lipomas follow fat 
signal on all MRI sequences. MRI readily demonstrates features suggesting malignancy and is the preferred diagnostic imaging study for the evaluation of soft tissue masses. ${ }^{3,4}$ Some lipomatous lesions demonstrate internal complexity such as thickened septations or nodularity while lacking clearly malignant features and may be classified as an atypical lipomatous tumor. ${ }^{5}$ On the other hand, some fatty lesions may be difficult to distinguish from surrounding subcutaneous fat. Such palpable simple fatty lesions lacking a clear definable capsule on MRI are often reported as nonencapsulated lipoma. ${ }^{6}$ Liposarcoma and atypical lipomatous tumor are typically treated with excision, while most lipomas do not require treatment unless there are mechanical symptoms or cosmetic indications for removal. ${ }^{7}$

Lipomas most commonly arise in the trunk or proximal extremities, while lesions in the hands and feet are much less common. ${ }^{2}$ Several reported cases in the literature describe lesions in the foot and ankle. ${ }^{8-15}$ In our practice, we have noticed that subcutaneous fat along the medial plantar margin of the midfoot between the abductor hallucis and flexor digitorum brevis muscles often has a pattern resembling a discrete lipoma. In some cases, well-defined fat containing regions with this appearance, in the setting of symptoms, has led to surgical intervention. Lui describes an endoscopic resection of a lipoma from the medial midfoot at the location we commonly see this finding. ${ }^{9}$ Taweel and Raikin describe resection of a similar "heel lipoma" at the same location. ${ }^{10}$ Given the striking similarity reported in the medical literature to what we have observed as a common incidental finding, we sought to understand whether a fatty pseudolesion may occur at this specific location.

\section{Methods}

To analyze the prevalence of a fatty pseudolesion at the medial midfoot, ankle MRI examinations performed sequentially over a 3-month period at our institution were retrospectively reviewed. The proposed retrospective study design was reviewed by the Institutional Review Board and granted exempt status.

Ankle MRI examinations were chosen for evaluation as they are obtained with a dedicated ankle coil and routinely include the medial midfoot in the field of view. The standard protocol at our institution includes $\mathrm{T} 1$ and $\mathrm{T} 2$ fat-saturated images in the axial, coronal, and sagittal planes.

Ninety-eight examinations performed on patients between ages of 18 and 89 between February 1, 2019 and May 1, 2019 were identified. Examinations were excluded if there was a history of surgery, amputation, or active infection at the medial midfoot site of interest. If more than one examination for any given patient was identified in this time frame, only the more recent MRI examination was included. MRI examinations were also excluded if they were nondiagnostic in quality due to motion or hardware artifact ( - Fig. 1 ).

Following exclusion, 91 cases remained for evaluation. Each of the 91 MRI examinations was evaluated separately in a blinded manner by three attending musculoskeletal radiologists (initials blinded) with 10,4 , and 1 years of

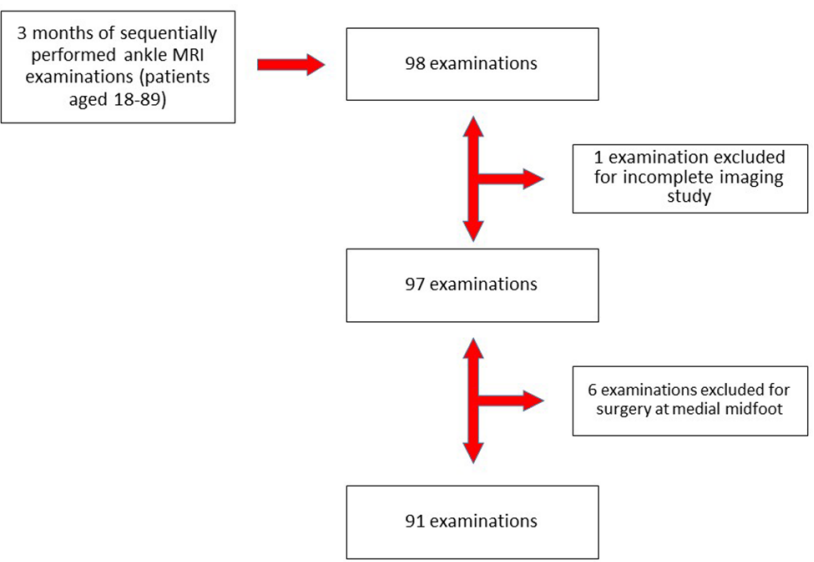

Fig. 1 Inclusion criteria for ankle magnetic resonance imaging (MRI) examinations.

musculoskeletal radiology experience, respectively. Patient age and sex were recorded. For each case, all indications for the study were recorded including mass, fracture, plantar fasciitis, instability, impingement, and tendinosis.

For each study, readers were instructed to evaluate the medial midfoot and identify the largest region of uninterrupted subcutaneous fat. Measurements were then made in this region by placing the longest caliper tool possible while staying within fat that was free of any low signal reticulations. Measurements were recorded in three axes (axial, coronal craniocaudal, and coronal transverse). The long axis (axial) measurement was made on T1-weighted axial images ( - Fig. 2A and C), while coronal craniocaudal and coronal transverse measurements were made on coronal T1-weighted images ( $\boldsymbol{- F i g . ~ 2 B}$ and $\mathbf{D}$ ). These measurements were recorded and analyzed without regard to the radiologist's personal assessment as to the presence or absence of a pseudolesion. Measurements completed for each of the three readers did not directly determine the presence or absence of any discrete lesion.

Initial data collection provided only measurements without attempt at defining whether a pseudolesion was present. A secondary analysis was therefore performed on the measurement data during which cases were classified into categories of "present," "indeterminate," and "absent" based on sliding scale of size cutoffs. Cases were classified as "pseudolesion present" at a given cutoff when the measured region of fat exceeded the cutoff size in all dimensions by at least two of three readers. Conversely, cases were classified as "pseudolesion absent" when the measured fat region was less than the cutoff in all dimensions by at least two of three readers. The remaining cases were classified as indeterminate.

Means and standard deviations are reported for continuous variables, whereas counts and percentages are reported for categorical variables. Intraclass correlation (ICC) estimates and their 95\% confidence intervals (CIs) were calculated based on a single-rating, consistency, two-way mixed effects model. Two multivariate linear regression models were performed to evaluate whether the "pseudolesion" size is associated with age or sex. The primary effect assessed was the interaction between the dimension (axial, coronal 

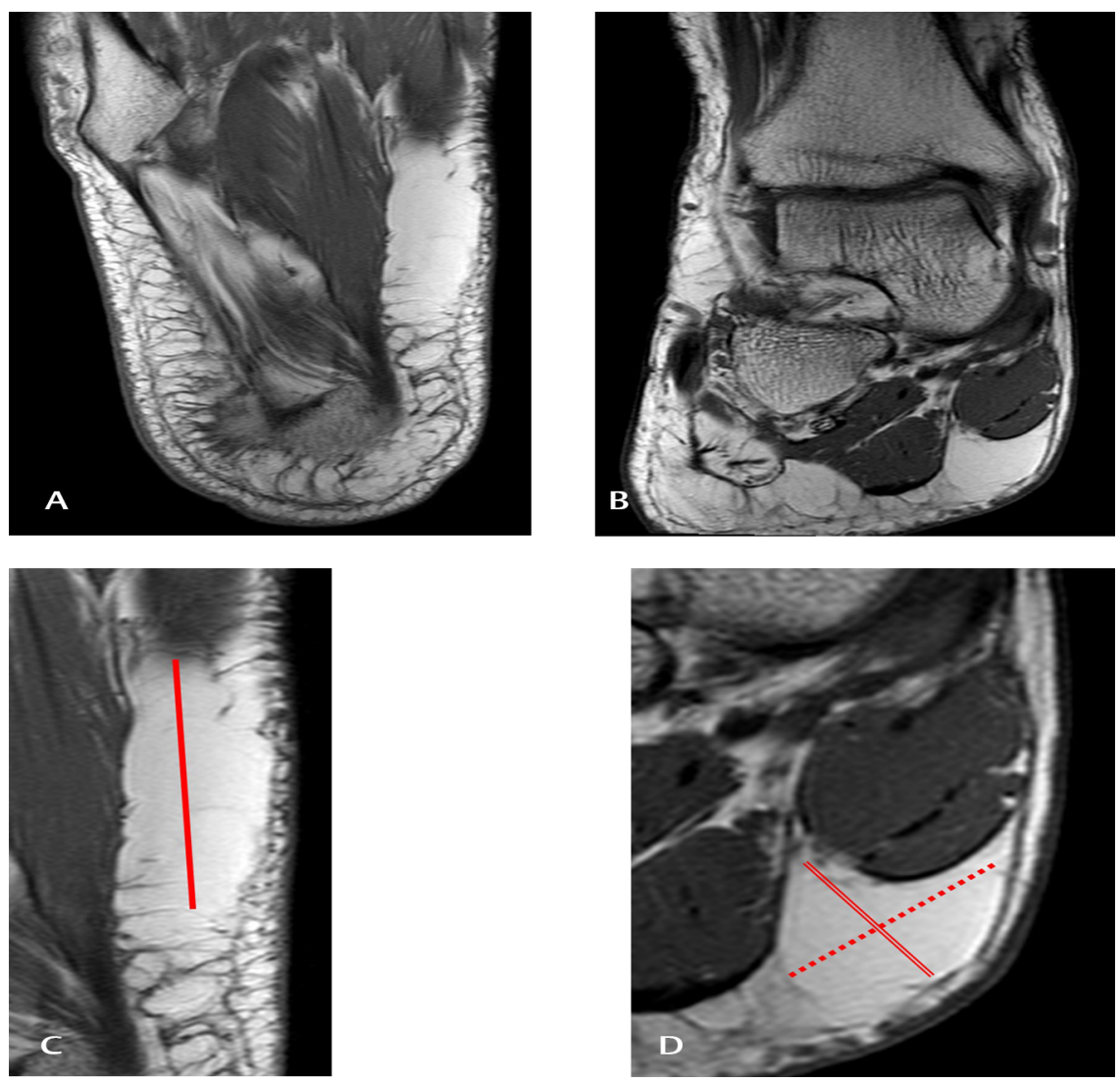

Fig. 2 Example case demonstrating lack of low signal reticulations in subcutaneous fat giving the vague impression of a mass or "pseudolesion." T1-weighted images in the axial (A) and coronal (B) planes demonstrate the area of interest. An axial measurement (red line) is shown in (C), while the coronal craniocaudal (double red line) and coronal transverse (dotted red line) are shown in (D).

craniocaudal, coronal transverse) and either age or sex, while adjusting for the reader effect and accounting for both the within-patient correlation and within-reader correlation using a Kronecker product. Statistical significance was set at $p<0.05$. Descriptive statistics, ICC, and figures were compiled in $\mathrm{R}^{16}$ and statistical modeling was conducted in SAS 9.4 software (SAS Institute Inc., Cary, North Carolina, United States).

\section{Results}

Ninety-one ankle MRI examinations were evaluated individually by three musculoskeletal radiologists. Thirty-seven male and 54 female patients were included in the cohort that had a mean age of 48 years. Indications included a variety of common diagnoses involving the foot and ankle. No indication was provided for five examinations and multiple indications were included for nine examinations. Descriptive statistics for the 91 examinations evaluated are included in - Table 1. Of note, only six cases specified "mass" in the indication and none of these specified the medial midfoot region as the area of interest. Pain was the most common indication and was listed in $44 \%$ of evaluated cases.

Measurements of the largest area of subcutaneous fat that was free of low signal reticulations yielded some cases with large apparent mass-like regions of subcutaneous fat reminiscent of a lipoma, while others had numerous reticular low
Table 1 Characteristics of included MRI examinations

\begin{tabular}{|l|l|}
\hline Age & $\begin{array}{l}\text { Overall } \\
(\boldsymbol{n}=91)\end{array}$ \\
\hline Mean (SD) & \\
\hline Sex & $48.4(15.9)$ \\
\hline Male & \\
\hline Female & $37(40.7 \%)$ \\
\hline Indications & $54(59.3 \%)$ \\
\hline Achilles & \\
\hline Fracture or stress fracture & $8(8.8 \%)$ \\
\hline Impingement & $6(6.6 \%)$ \\
\hline Instability & $3(3.3 \%)$ \\
\hline Mass & $2(2.2 \%)$ \\
\hline Multiple & $6(6.6 \%)$ \\
\hline No indication & $9(9.9 \%)$ \\
\hline Pain & $4(4.4 \%)$ \\
\hline Plantar fasciitis & $40(44.0 \%)$ \\
\hline Tendinosis & $3(3.3 \%)$ \\
\hline
\end{tabular}

Abbreviations: MRI, magnetic resonance imaging; SD, standard deviation. 

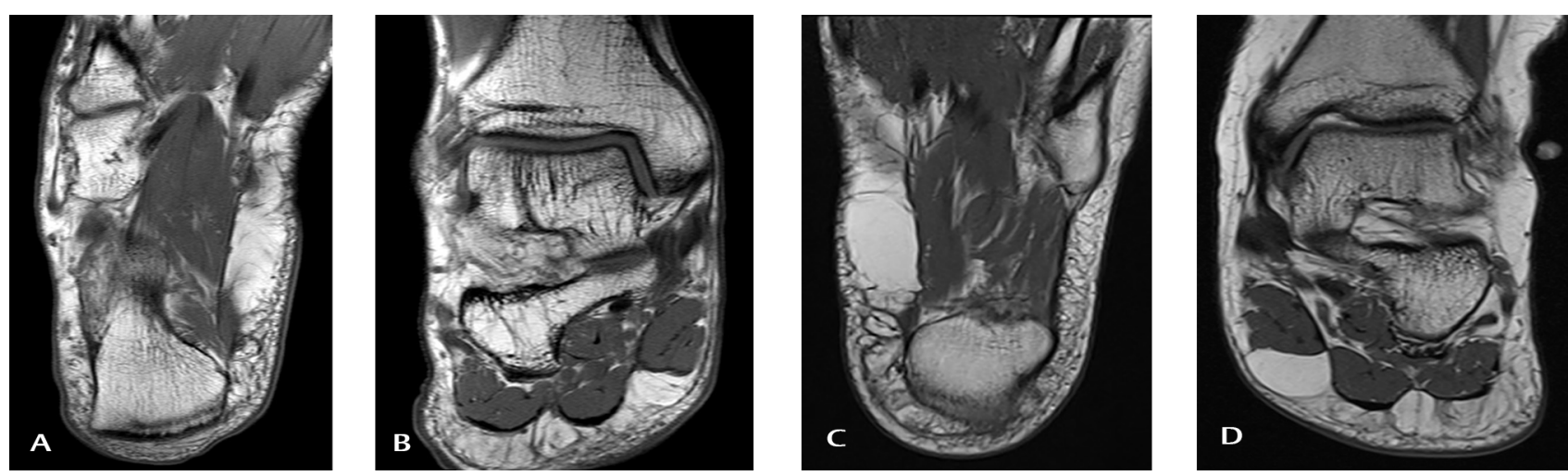

Fig. 3 Examples of cases demonstrating variability of subcutaneous fat at medial midfoot. Note in the example axial (A) and coronal (B) T1-weighted images of the same patient the absence of a mass-like region of subcutaneous fat free of low signal reticulations. Axial (C) and coronal (D) T1-weighted images in another patient showing prominent fat in the same region reminiscent of a lipoma.

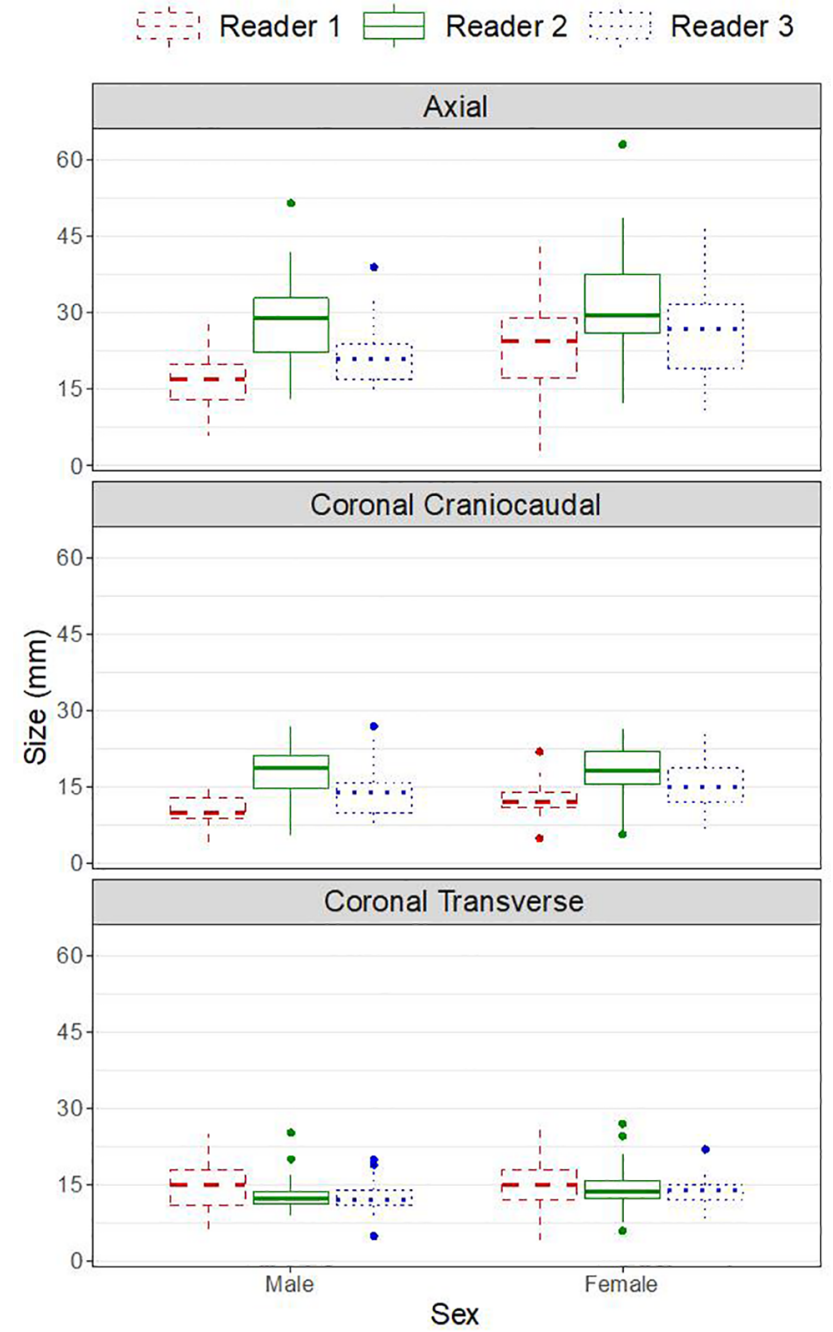

Fig. 4 Lesion measurements by reader and patient sex in the axial, coronal craniocaudal, and coronal transverse dimensions.

signal reticulations such that the medial midfoot fat resembled that found elsewhere about the ankle in the field of view (-Fig. 3).

After accounting for potential measurement correlations (i.e., 3 dimensions and 3 readers per subject) and the reader
- Reader $1-$ Reader $2 \cdots$ Reader 3
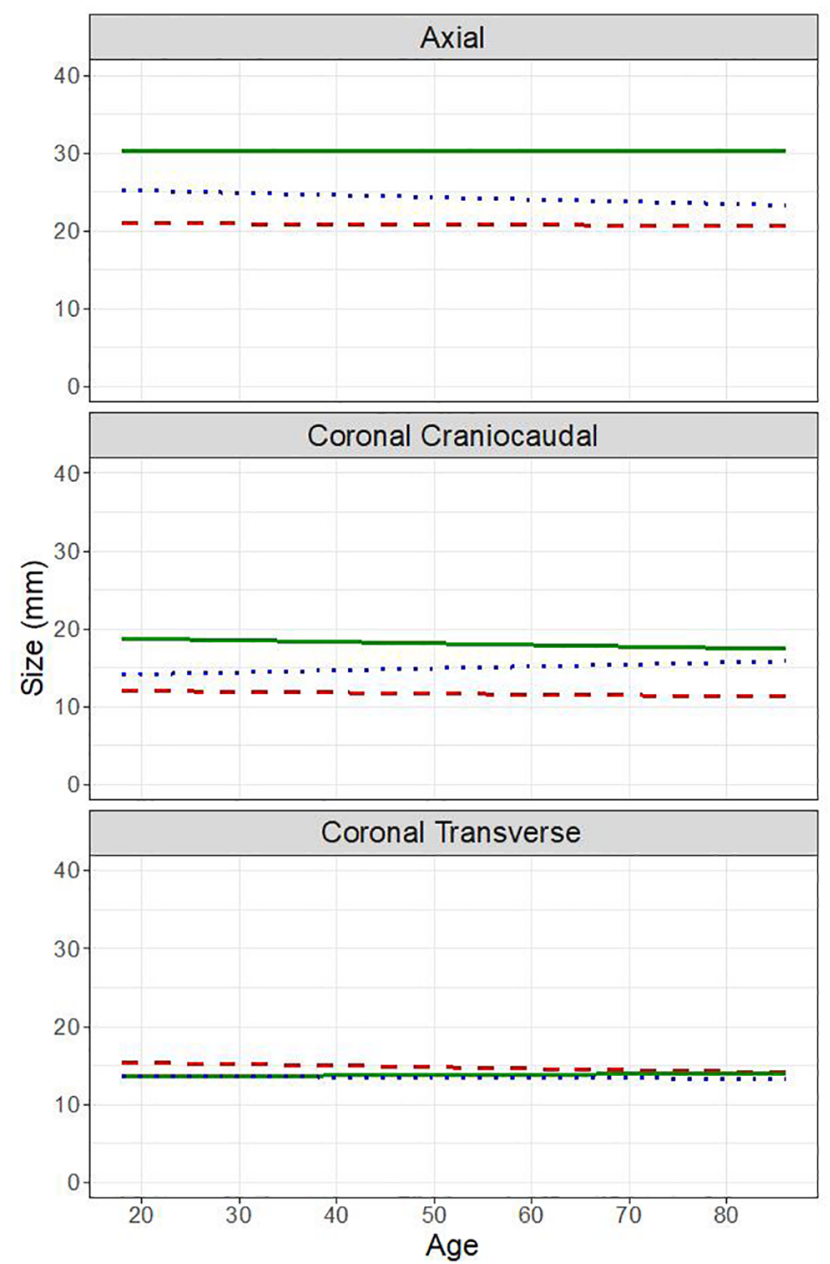

Fig. 5 Lesion measurements (represented by least-squares regression means) by size in the axial, coronal craniocaudal, and coronal transverse dimensions compared with patient age showing no significant trend toward increasing or decreasing lesion size.

effect, females had significantly larger "lesion" sizes compared with males in the axial dimension $(95 \% \mathrm{CI}=2.5 \mathrm{~mm}$ to $7.3 \mathrm{~mm}, p<0.01$ ), but sex was not significantly different 
in the coronal craniocaudal $(95 \% \mathrm{CI}=-0.2 \mathrm{~mm}$ to $2.8 \mathrm{~mm}$, $p=0.09)$ or coronal transverse dimension $(95 \% \mathrm{CI}=-0.4 \mathrm{~mm}$ to $2.1 \mathrm{~mm}, p=0.18$; - Fig. 4 ).

After accounting for potential measurement correlations (i.e., 3 dimensions and 3 readers per subject) and effect of the reader and dimension, age was not associated with size $(p=0.79)$ (-Fig. 5).

Reader intraclass correlation (ICC) coefficients for individual measurements in the axial, coronal craniocaudal, and coronal transverse dimensions were 0.68 (95\% CI $=0.58$, $0.76), 0.42(95 \% \mathrm{CI}=0.29,0.54)$, and $0.25(95 \% \mathrm{CI}=0.12,0.39)$, respectively.

Cases classified as present or absent by at least two of three readers were documented with remaining cases classified as indeterminate. The results of the secondary analysis using a sliding scale of potential cutoff values are displayed in - Table 2, which give a sense of pseudolesion sizes encountered in the sample.

Seventy-nine of 91 cases demonstrated a region of fat $\geq$ $10 \mathrm{~mm}$ in all dimensions that was free of internal low signal reticulations, as measured by at least two of three readers. If the size criteria are increased to at least $15 \mathrm{~mm}$, the number of cases with a "pseudolesion" present drops to 13 . When the size criteria are increased at least $18 \mathrm{~mm}$, no "psuedolesion" cases would be considered present.

Conversely, when defining an absent case, only two cases had measurements $<15 \mathrm{~mm}$ in all dimensions as measured by at least two of three readers. When the size criteria are shifted down to $<13 \mathrm{~mm}$, no "absent" cases were found.

\section{Discussion}

It is evident that in even a relatively small sample such as the one presented in this study, it is common to encounter a mass-like pseudolesion at the medial midfoot. Despite the fact that the midfoot could not be biopsied for pathologic confirmation in this series, the very high prevalence of focal mass-like fat deposition at this specific region is worthy of note and fits with our anecdotal experience that subcutaneous fat at this location can resemble a lipoma. While this may be commonly known to musculoskeletal radiologists, it may not be well known to all providers who may encounter such imaging studies.

Measurements of mass-like fat at the medial midfoot by three independent radiologists yielded a high degree of variability as reflected by the low reader ICC coefficients for measurements in all planes. This makes intuitive sense as we assume that no true mass was present in these cases. It is, however, notable that in 79 of 91 cases, a focal mass-like region of fat measuring at least $10 \mathrm{~mm}$ in all orthogonal dimensions could be perceived by at least two of three readers. These data demonstrate that a region of fat resembling at least a small lipoma is visible in a substantial number of cases.

Using a larger size cutoff of $15 \mathrm{~mm}$ in all dimensions still resulted in at least two of three readers perceiving a pseudolesion in $14 \%$ of cases (13 of 91). At a cutoff of $18 \mathrm{~mm}$ (or $20 \mathrm{~mm}$ as a "close enough" round number), no mass-like fatty pseudolesions were identified. While a large sample size may reveal cases that exceed this value, our findings suggest

Table 2 Classification results for secondary analysis using sliding scale cutoffs of the original measurements

\begin{tabular}{|c|c|c|c|}
\hline Size cutoff (mm) & Present & Absent & Indeterminate \\
\hline 10 & 79 & 0 & 12 \\
\hline 11 & 64 & 0 & 27 \\
\hline 12 & 51 & 0 & 40 \\
\hline 13 & 34 & 0 & 57 \\
\hline 14 & 23 & 1 & 67 \\
\hline 15 & 13 & 2 & 76 \\
\hline 16 & 5 & 7 & 79 \\
\hline 17 & 3 & 7 & 81 \\
\hline 18 & 0 & 12 & 79 \\
\hline 19 & 0 & 17 & 74 \\
\hline 20 & 0 & 23 & 68 \\
\hline 21 & 0 & 30 & 61 \\
\hline 22 & 0 & 36 & 55 \\
\hline 23 & 0 & 41 & 50 \\
\hline 24 & 0 & 42 & 49 \\
\hline 25 & 0 & 45 & 46 \\
\hline 26 & 0 & 50 & 41 \\
\hline 27 & 0 & 55 & 36 \\
\hline 28 & 0 & 64 & 27 \\
\hline 29 & 0 & 66 & 25 \\
\hline 30 & 0 & 70 & 21 \\
\hline
\end{tabular}


that the range of normal nonseptated fat in this region is in almost all cases less than $2 \mathrm{~cm}$.

It is interesting to note that despite increasing adiposity throughout the body seen as a part of normal aging, ${ }^{17}$ there was no increased prevalence of a fatty pseudolesion at the medial midfoot with advanced age in this cohort.

Lipomas are commonly encountered lesions and rarely present a diagnostic dilemma. In some cases, they may be associated with pain, mechanical symptoms, or cosmetic deformity requiring resection. ${ }^{18}$ Imaging studies such as MRI are excellent at demonstrating the margins of such lesions and aiding in surgical planning, when indicated. In some cases, however, it may be difficult to determine whether a lipoma is present at a location of symptoms. ${ }^{19}$

Imaging characterization of any potential mass requires documentation of additional secondary features such as location, displacement or invasion of adjacent structures, and reaction of surrounding tissues. Correlation for pain or a palpable mass is also clinically important when the imaging features are worrisome. Keeping this approach in mind should help providers to more appropriately evaluate the probability of a truly problematic lesion and avoid misdiagnosis that could potentially subject a patient to unnecessary pain, deformity, and expense.

Fatty masses at the medial midfoot are reported in the literature, some of which have been resected with reported improvement in patient symptoms. ${ }^{9,10}$ Given the prevalence of a fatty pseudolesion at this location, one wonders if some resected "lipomas" truly represented masses or variant fat. It is interesting to note the clinical recovery of a patient for whom surgical removal of a purported lipoma at the medial midfoot was performed..$^{10}$ While it is certainly possible that a true discrete lesion was present at this location in these published reports, it is also possible that decreased mass effect from normal subcutaneous fat, restricted use of the foot during recovery, or spontaneous resolution of symptoms may also have played a role in the reported positive clinical outcome.

This study has several limitations. Ninety-one MRI examinations were ultimately included and gathering a larger cohort would allow for a more precise statistical description of a common fatty pseudolesion along the medial midfoot; however, the utility would be limited in that awareness of the variant appearance is more important than the specifics of its dimensions. Also, no pathologic confirmation was obtained, though this would be impractical for normal asymptomatic fat in patients imaged for other reasons.

In light of existing literature describing resection of fatty masses at the medial midfoot, it is important for radiologists and surgeons to recognize the characteristic appearance of fat at the medial midfoot to prevent unneeded surgery or other treatments. Awareness of the high prevalence of prominent fat at this location should help focus clinical and imaging attention on other likely pathologic causes of pain or swelling.

\section{Note}

The manuscript has not been orally presented

\section{Funding}

This research did not receive any specific grant from funding agencies in the public, commercial, or not-for-profit sectors.

\section{Conflicts of Interest}

This publication was supported by National Institutes of Health/National Center for Research Resources (NIH/NCRR) blinded. Its contents are the authors' sole responsibility and do not necessarily represent official NIH views.

\section{Acknowledgment}

Study data were collected and managed using Research Electronic Data Capture (REDCap) electronic data capture tools hosted at the blinded. ${ }^{20}$ REDCap is a secure, web-based application designed to support data capture for research studies, providing (1) an intuitive interface for validated data entry, (2) audit trails for tracking data manipulation and export procedures, (3) automated export procedures for seamless data downloads to common statistical packages, and (4) procedures for importing data from external sources.

\section{References}

1 Murphey MD, Carroll JF, Flemming DJ, Pope TL, Gannon FH, Kransdorf MJ. From the archives of the AFIP: benign musculoskeletal lipomatous lesions. Radiographics 2004;24(5):1433-1466

2 Kransdorf MJ. Benign soft-tissue tumors in a large referral population: distribution of specific diagnoses by age, sex, and location. AJR Am J Roentgenol 1995;164(2):395-402

3 Drevelegas A, Pilavaki M, Chourmouzi D. Lipomatous tumors of soft tissue: MR appearance with histological correlation. Eur J Radiol 2004;50(3):257-267

4 Rougraff BT, Durbin M, Lawerence J, Buckwalter K. Histologic correlation with magnetic resonance imaging for benign and malignant lipomatous masses. Sarcoma 1997;1(3-4):175-179

5 Gupta P, Potti TA, Wuertzer SD, Lenchik L, Pacholke DA. Spectrum of fat-containing soft-tissue masses at MR imaging: the common, the uncommon, the characteristic, and the sometimes confusing. Radiographics 2016;36(3):753-766

6 Roberts CC, Liu PT, Colby TV. Encapsulated versus nonencapsulated superficial fatty masses: a proposed MR imaging classification. AJR Am J Roentgenol 2003;180(5):1419-1422

7 Crago AM, Singer S. Clinical and molecular approaches to well differentiated and dedifferentiated liposarcoma. Curr Opin Oncol 2011;23(4):373-378

8 Azevedo CP, Casanova JM, Guerra MG, Santos AL, Portela MI, Tavares PF. Tumors of the foot and ankle: a single-institution experience. J Foot Ankle Surg 2013;52(2):147-152

9 Lui TH. Endoscopic resection of subcutaneous lipoma and tumor-like lesion of the foot. Foot 2016;26:36-40

10 Taweel NR, Raikin SM. Heel lipoma mimicking plantar fasciitis in a ballroom dancer. J Foot Ankle Surg 2015;54(2):251-253

11 Berlin SJ, Mirkin GS, Tubridy SP. Tumors of the heel. Clin Podiatr Med Surg 1990;7(2):307-321

$12 \mathrm{Ng} \mathrm{A}$, Beegle T, Rockett AK. Atypical presentation of plantar fasciitis secondary to soft-tissue mass infiltration. J Am Podiatr Med Assoc 2001;91(2):89-92

13 Satish C. A rare case of dumb-bell lipoma of the sole. J Plast Reconstr Aesthet Surg 2011;64(12):e329-e331 
14 Olaoye IO, Adesina MD, Solitary massive lipoma in the planter aspect of great toe presenting as two masses SAGE Open Med Case Rep, 2017 5:p. $2050313 X 17730265$

15 Math KR, Pavlov H, DiCarlo E, Bohne WH. Spindle cell lipoma of the foot: a case report and literature review. Foot Ankle Int 1995;16(4):220-226

16 R Core Team. (2020). R: A language and environment for statistical computing. R Foundation for Statistical Computing, Vienna, Austria. URL https://www.R-project.org/. Accessed June 29, 2021

17 Reinders I, Visser M, Schaap L. Body weight and body composition in old age and their relationship with frailty. Curr Opin Clin Nutr Metab Care 2017;20(1):11-15
18 Elia S, Cerioli A, Fiaschetti V, Granai AV. Infraclavicular subpectoral lipoma causing thoracic outlet syndrome. Int J Surg Case Rep 2015;9:101-104

19 Yamamoto A, Kikuchi Y, Kusakabe T, et al. Imaging spectrum of abnormal subcutaneous and visceral fat distribution. Insights Imaging 2020;11(1):24

20 Harris PA, Taylor R, Thielke R, Payne J, Gonzalez N, Conde JG. Research electronic data capture (REDCap)-a metadata-driven methodology and workflow process for providing translational research informatics support. J Biomed Inform 2009;42(2):377-381 